\title{
A Systematically Assembled Signature of Genes to be Deep-Sequenced for Their Associations with the Blood Pressure Response to Exercise
}

\author{
Linda S. Pescatello ${ }^{1,2, *}$, Paul Parducci ${ }^{1}$, Jill Livingston ${ }^{3}$ and Beth A. Taylor ${ }^{1,2,4}$ \\ 1 Department of Kinesiology, University of Connecticut, Storrs, CT 06269, USA; \\ Paul.Parducci@uconn.edu (P.P.); Beth.Taylor@uconn.edu (B.A.T.) \\ 2 Institute for Systems Genomics, University of Connecticut, Storrs, CT 06269, USA \\ 3 Homer Babbidge Library, Health Sciences, University of Connecticut, Storrs, CT 06269, USA; \\ Jill.Livingston@uconn.edu \\ 4 Preventive Cardiology, Hartford Hospital, Hartford, CT 06269, USA \\ * Correspondence: Linda.Pescatello@uconn.edu; Tel.: +1-860-486-0008; Fax: +1-860-486-1123
}

Received: 9 March 2019; Accepted: 4 April 2019; Published: 11 April 2019

\begin{abstract}
Background: Exercise is one of the best nonpharmacologic therapies to treat hypertension. The blood pressure (BP) response to exercise is heritable. Yet, the genetic basis for the antihypertensive effects of exercise remains elusive. Methods: To assemble a prioritized gene signature, we performed a systematic review with a series of Boolean searches in PubMed (including Medline) from earliest coverage. The inclusion criteria were human genes in major BP regulatory pathways reported to be associated with: (1) the BP response to exercise; (2) hypertension in genome-wide association studies (GWAS); (3) the BP response to pharmacotherapy; (4a) physical activity and/or obesity in GWAS; and (4b) BP, physical activity, and/or obesity in non-GWAS. Included GWAS reports disclosed the statistically significant thresholds used for multiple testing. Results: The search yielded 1422 reports. Of these, 57 trials qualified from which we extracted 11 genes under criteria 1, 18 genes under criteria 2, 28 genes under criteria 3, 27 genes under criteria 4a, and 29 genes under criteria 4b. We also included 41 genes identified from our previous work. Conclusions: Deep-sequencing the exons of this systematically assembled signature of genes represents a cost and time efficient approach to investigate the genomic basis for the antihypertensive effects of exercise.
\end{abstract}

Keywords: antihypertensive therapy; healthy lifestyle; physical exercise

\section{Introduction}

Cardiovascular disease (CVD) is the leading cause of death worldwide, claiming 31\% (17.5 million) of deaths globally [1,2]. Hypertension is the most common, costly, and preventable CVD risk factor [1]. The American College of Cardiology (ACC)/American Heart Association (AHA) recently redefined hypertension to a lower blood pressure (BP) threshold of $130 \mathrm{mmHg}$ for systolic BP (SBP) or $80 \mathrm{mmHg}$ for diastolic BP (DBP) [3]. This change has been met with some opposition. Nonetheless, the lower BP thresholds now classify $50 \%$ of adults in the United States with hypertension [1], underscoring the importance of hypertension as a public health problem.

The authors of the AHA/ACC report rated exercise as one of the best nonpharmacologic therapies to treat hypertension because aerobic exercise training lowers BP 5-8 $\mathrm{mmHg}$ among adults with hypertension [3]. The magnitude of these BP reductions rival those that result from taking antihypertensive medication [4,5], may lower the risk of CVD risk by 4-22\% and stroke by 6-41\% [6-8], and reduce the resting BP of some adults with hypertension into normal ranges [9,10]. Accordingly, 
professional organizations from around the world recommend adults with hypertension participate in $30-60 \mathrm{~min} /$ day of aerobic exercise, such as walking or jogging, on most days of the week [5,11].

Despite the well-documented antihypertensive benefits of exercise, many adults with hypertension do not exercise to lower their BP [12,13]. Reasons for this non-adherence are varied; however, one reason relevant to the topic of this systematic review is that there is significant inter-individual variability in the $\mathrm{BP}$ response to exercise partially attributed to genetic predispositions that led some to believe exercise does not work as antihypertensive therapy [14,15]. Indeed, investigators from the HEalth, RIsk Factors Exercise TrAining and GEnetics or HERITAGE Family Study involving over 700 subjects established the BP response to aerobic exercise is heritable $(h=0.13-0.42)[14,16-19]$. Nearly 20 years ago this discovery prompted our laboratory group $[20-24]$ and others $[16-19,25]$ to conduct candidate genes association studies to identify genetic variants that account for a clinically meaningful proportion of the variability in the BP response to exercise. However, these efforts have met with little success $[17,25,26]$.

More recently, we exploited advances in genomic technology that emerged since our discovery phase candidate gene association studies as well as other strategies to bolster our statistical power to detect genetic variants associated with the BP response to exercise in a replication cohort of subjects with hypertension whose characteristics resembled those from our earlier studies. In this series of studies, we deep-sequenced the exons of genes using the Illumina TruSeq Custom Amplicon kit (Catalog\# FC-130-1001, Illumina, San Diego, CA, USA) on a prioritized signature of 41 BP and exercise genes that contained genes identified from our prior work in addition to those obtained from a systematic review of the literature of genes reported to be associated with hypertension or the BP response to exercise or pharmacotherapy [27-29]. After adjustment for multiple testing, despite the small sample size, we found variants in $61 \%$ of the 41 genes in the prioritized panel associated with the BP response to exercise. We attributed the high proportion of the significant BP-genotype associations that we found to a focused inquiry of variants with a gene signature obtained from a systematic review of the literature that reduced the search space within the genome; and the use of high throughput exon sequencing to concentrate on functional gene regions and standardized BP and exercise protocols that were well controlled and closely supervised.

The purpose of this systematic review is to update and expand our original systematic review to assemble a prioritized signature of BP and exercise genes whose exons can then be deep-sequenced among a larger, more ethnically and gender diverse sample of adults that were reported in the qualifying studies to have hypertension to better inform the genomic basis for the antihypertensive effects of exercise. The long-term goal of this work is to develop personalized exercise prescriptions based upon genetic predispositions and other clinical characteristics to optimize the BP benefits of exercise.

\section{Systematic Review Methods}

This systematic review followed the specifications of the Preferred Reporting Items for Systematic Reviews and Meta-Analyses (PRISMA) statement [30,31]. A series of comprehensive Boolean searches were run in PubMed (including Medline). The first search replicated the original search done by Bruneau et al. [25] in the four databases of Medline, Biosis, Scopus, and Web of Science and included contiguous dates. The additional three searches were run from earliest coverage to 11 May 2017 to locate trials that met our predetermined inclusion criteria. These a priori criteria were human genes in major $\mathrm{BP}$ regulatory pathways reported to be associated with: (1) the BP response to exercise; (2) hypertension in genome-wide association studies (GWAS); (3) the BP response to pharmacotherapy; (4a) physical activity and/or obesity, a major risk factor for hypertension as $80 \%$ of adults with hypertension are overweight to obese [32] in GWAS; and (4b) BP, physical activity, and/or obesity in non-GWAS from relevant reference searches of the authors' files. We also included the genes from the prioritized signature of BP and exercise genes from our previous work [27-29]. 
The major regulatory BP pathways that a qualifying gene could be in were the renin angiotensin aldosterone system, endothelial nitric oxide synthase pathway, and/or the sympathetic nervous system, and/or pathways involved with vascular function and structure, fluid and electrolyte control, inflammation, insulin sensitivity, lipid metabolism, and obesity. Of note, many of the GWAS we initially located as indicating they involved genes associated with hypertension upon closer scrutiny involved genes associated with resting BP rather than hypertension per se so that they were excluded. In all GWAS for the gene to be included on the panel, the reported associations with the BP response to exercise must have met preestablished statistically significant thresholds for multiple testing which is commonly set at $p=5 \times 10^{-8}$. The full search strategy for each of the four a priori criteria are depicted in Tables 1-4.

Table 1. Criteria 1: Genes $(n=11)$ in major blood pressure regulatory pathways associated with the blood pressure response to exercise. Two searches were conducted on 05/11/2017 and a total of 321 records were identified.

(2006/01:2017/06 [edat]) AND ("mean arterial" OR "blood pressure"[mesh] OR "blood pressure" OR "blood pressures" OR "arterial pressure" OR "arterial pressures" OR hypertension OR hypotension OR normotension OR hypertensive OR hypotensive OR normotensive OR "systolic pressure" OR "diastolic pressure" OR "pulse pressure" OR "venous pressure" OR "pressure monitor" OR hypotension OR "pre hypertension" OR "bp response" OR "bp decrease" OR "bp reduction" OR "bp monitor" OR "bp monitors" OR "bp measurement") AND ("exercise" [mesh] OR exercise OR exercises OR running[mesh] OR "bicycle" OR "bicycles" OR "bicycling" OR walking[mesh] OR treadmill* OR "weight lifting" OR "weight training" OR "weight bearing" OR "resistance training" OR "strength training" OR "endurance training" OR "speed training" OR "training duration" OR "training frequency" OR "training intensity" OR "aerobic endurance") AND ("randomized controlled trial" [pt] OR "nonrandomized controlled" OR "nonrandomized control" OR controlled clinical trial[pt] OR "randomized controlled trial"[publication type] OR random allocation[mh] OR clinical trial[pt] OR "comparative study" OR "comparative studies" OR clinical trials[mh] OR "clinical trial" $[\mathrm{tw}]$ OR “latin square" [tw] OR random*[tw] OR research design[mh:noexp] OR "comparative study"[publication type] OR "evaluation studies"[publication type] OR "prospective studies"[mh] OR "cross-over studies"[mh] OR "control"[tw] OR "controlled"[tw]) AND ("gene" OR "genes" OR "genotype" OR "genotypes" OR "snp" OR polymorphism* OR "DNA" OR "minor allele" OR "minor alleles" OR "single nucleotide polymorphism" OR "single nucleotides polymorphisms" OR genetic*) NOT ("DASH"[tiab] OR "cancer" OR "neoplasms" OR "review" [pt] OR "fibromyalgia" OR "alzheimers" OR "alzheimer" OR "pregnant" OR "pregnancy" OR “obesity/drug therapy"[mesh] OR "diet therapy"[mesh] OR "diet therapy" [subheading] OR "caffeine" OR "eating change" OR "activities of daily living" OR "dehydration" OR "dehydrate" OR "dehydrated" OR "dietary salt" OR "epilepsy" OR “influenza" OR "flu" OR "pneumonia" OR "septicemia" OR "hiv" OR "Acquired Immunodeficiency Syndrome" OR "meningitis" OR "substance abuse" OR "alcoholism" OR "drug abuse" OR "Cross-Sectional Studies"[MeSH Terms] OR "Prospective Studies"[MeSH Terms] OR “epidemiology"[Subheading]).

("mean arterial" OR "blood pressure"[mesh] OR "blood pressure" OR "blood pressures" OR "arterial pressure" OR "arterial pressures" OR hypertension OR hypotension OR normotension OR hypertensive OR hypotensive OR normotensive OR "systolic pressure" OR "diastolic pressure" OR "pulse pressure" OR "venous pressure" OR "pressure monitor" OR hypotension OR "pre hypertension" OR "bp response" OR "bp decrease" OR "bp reduction" OR "bp monitor" OR "bp monitors" OR "bp measurement") AND ("ambulatory blood pressure" OR "exercise" [mesh] OR exercise[ti] OR exercises OR running[mesh] OR running[ti] OR "bicycle" OR "bicycles" OR “bicycling” OR walking[mesh] OR walking[ti] OR treadmill* OR "weight lifting" OR "weight training" OR "weight bearing" OR "resistance training" OR "strength training" OR "endurance training" OR "speed training" OR "training duration" OR "training frequency" OR "training intensity" OR "aerobic endurance" OR "aerobic training") AND (gwa[ti] OR gwas[ti] OR genome[ti] OR "gene"[ti] OR "genes"[ti] OR "genotype"[ti] OR "genotypes"[ti] OR "genotyping"[ti] OR "snp" [ti] OR

Search 2: "snps"[ti] OR polymorphism*[ti] OR "DNA"[ti] OR allele[ti] OR alleles[ti] OR "minor allele" OR "minor alleles" OR "single nucleotide polymorphism" OR "single nucleotides polymorphisms" OR genetic*[ti] OR "trait locus"[ti] OR "loci"[ti] OR "Genetic Predisposition to Disease"[MeSH] OR "Genotype"[MeSH] OR "Gene Frequency"[MeSH] OR “Polymorphism, Single Nucleotide/genetics"[MESH] OR "Polymorphism, Single Nucleotide"[MAJR] OR “Genetic Loci”[Mesh] OR “Genetic Association Studies"[Mesh] OR "Genetic Variation"[Mesh]) NOT ("DASH"[tiab] OR "cancer" OR "neoplasms" OR "review"[pt] OR "fibromyalgia" OR "alzheimers" OR "alzheimer" OR "pregnant" OR "pregnancy" OR "obesity/drug therapy"[mesh] OR "diet therapy"[mesh] OR "diet therapy" [subheading] OR "caffeine" OR "eating change" OR "activities of daily living" OR "dehydration" OR "dehydrate" OR "dehydrated" OR "dietary salt" OR "epilepsy" OR "influenza" OR "flu" OR "pneumonia" OR "septicemia" OR "hiv" OR "Acquired Immunodeficiency Syndrome" OR "meningitis" OR "substance abuse" OR "alcoholism" OR "drug abuse" OR "Cross-Sectional Studies"[MeSH Terms] OR "Prospective Studies"[MeSH Terms] OR “epidemiology"[Subheading]). 
Table 2. Criteria 2: Genes $(n=18)$ in major blood pressure regulatory pathways associated with hypertension in genome wide association studies. The search was conducted on 05/11/2017 and a total of 188 records were identified.

\begin{abstract}
("mean arterial" OR "blood pressure"[mesh] OR "blood pressure" OR "blood pressures" OR "arterial pressure" OR "arterial pressures" OR hypertension OR hypotension OR normotension OR hypertensive OR hypotensive OR normotensive OR "systolic pressure" OR "diastolic pressure" OR "pulse pressure" OR "venous pressure" OR "pressure monitor" OR hypotension OR "pre hypertension" OR "bp response" OR "bp decrease" OR "bp reduction" OR "bp monitor" OR "bp monitors" OR "bp measurement" OR "Blood Pressure/genetics"[MeSH]) AND ("resting blood pressure" OR "resting BP" OR "exercise" [mesh] OR exercise[ti] OR exercises OR running[mesh] OR running[ti] OR "bicycle" OR "bicycles" OR "bicycling" OR walking OR walking[mesh] OR walking[ti] OR treadmill* OR "weight lifting" OR "weight training" OR "weight bearing" OR "resistance training" OR "strength training" OR "endurance training" OR "speed training" OR "training duration" OR "training frequency" OR "training intensity" OR "aerobic endurance" OR "aerobic training" OR "physical activity" OR "motor activity" [mesh] OR Overweight OR “Overweight”[Mesh] OR BMI OR “body mass" OR “Body Mass Index"[MeSH] OR “Waist Circumference" [MeSH] OR obesity OR "Obesity"[Mesh] OR obese) AND (gwa OR gwas OR "Genetic Association Studies"[Mesh] OR genomewide OR "genome wide" OR "genomewide association" OR "genome wide association" OR "genome-wide interaction") NOT ("review" [pt] OR “Cross-Sectional Studies"[MeSH Terms] OR Comment[pt] OR Editorial[pt] OR Letter[pt] OR "Case Reports"[pt] OR "case control"[ti] OR "case report" [ti] OR "case study"[ti] OR “case series"[ti] OR “Case-Control Studies"[Mesh] OR "Follow-Up Studies"[Mesh] OR "observational study" [ti] OR "prospective cohort" [ti] OR "cohort studies" [Mesh:NoExp] OR "cohort study"[ti] OR "Longitudinal Studies" [Mesh:NoExp] OR "Follow-Up Studies"[mesh] OR “Retrospective Studies"[mesh] OR "non-randomized"[ti] OR "follow up study"[ti] OR “Cross-Sectional Studies"[MeSH Terms] OR "Prospective Studies"[MeSH Terms] OR "epidemiology"[Subheading] OR "pulmonary hypertension" OR "pulmonary arterial hypertension" OR "heart transplant" OR "heart failure" OR "cystic fibrosis" OR "cancer" OR "neoplasms" OR "fibromyalgia" OR “alzheimers" OR "alzheimer" OR "pregnant" OR "pregnancy" OR "obesity/drug therapy"[mesh] OR "diet therapy" [mesh] OR "diet therapy" [subheading] OR "DASH"[tiab] OR meal[ti] OR "nutritional intervention" OR "dietary intervention" OR "nutritional counseling" OR "dietary counseling" OR "caffeine" OR "eating change" OR "activities of daily living" OR "dehydration" OR “dehydrate" OR “dehydrated" OR “dietary salt" OR sodium OR "epilepsy" OR "influenza" OR "flu" OR "pneumonia" OR "septicemia" OR arthritis OR "hiv" OR "Acquired Immunodeficiency Syndrome" OR "meningitis" OR "substance abuse" OR "alcoholism" OR "drug abuse" OR "spinal cord"[ti] OR "Sleep"[Majr] OR "Sleep Apnea Syndromes"[Majr] OR sleep[ti] OR contraceptive*[ti] OR (animals[mesh] NOT humans[mesh]) OR rat[ti] OR rats[ti] OR mouse[ti] OR mice[ti] OR pig[ti] OR pigs[ti] OR dog[ti] OR dogs[ti] OR canine[ti] OR cow[ti] OR cows[ti] OR bovine[ti]).
\end{abstract}

Table 3. Criteria 3. Genes $(n=28)$ in major blood pressure regulatory pathways associated with the blood pressure response to pharmacotherapy. The search was conducted on 05/11/2017 and a total of 711 records were identified.

\footnotetext{
("mean arterial" OR "blood pressure"[mesh] OR "blood pressure" OR "blood pressures" OR "arterial pressure" OR "arterial pressures" OR hypertension OR hypotension OR normotension OR hypertensive OR hypotensive OR normotensive OR "systolic pressure" OR "diastolic pressure" OR "pulse pressure" OR "venous pressure" OR "pressure monitor" OR hypotension OR "pre hypertension" OR "bp response" OR "bp decrease" OR "bp reduction" OR "bp monitor" OR "bp monitors" OR "bp measurement" OR "Blood Pressure/genetics"[Mesh]) AND ("Antihypertensive Agents"[Mesh] OR "Antihypertensive Agents" [Pharmacological Action] OR "anti-hypertensive agent" OR "antihypertensive agent" OR "anti hypertensive agent" OR "anti-hypertensive agents" OR "antihypertensive agents" OR "anti hypertensive agents" OR "anti-hypertensive drug" OR "antihypertensive drug" OR "anti hypertensive drug" OR "anti-hypertensive drugs" OR "antihypertensive drugs" OR "anti hypertensive drugs" OR "anti-hypertensive medication" OR "antihypertensive medication" OR "anti hypertensive medication" OR "anti-hypertensive medications" OR "antihypertensive medications" OR "anti hypertensive medications" OR "anti-hypertensives" OR antihypertensives OR "anti hypertensives" OR diuretic OR diuretics OR "Diuretics"[Mesh] OR acebutolol OR aliskiren OR Ambrisentan OR amlodipine OR atenolol OR "azilsartan medoxomil" OR benazepril OR betaxolol OR bisoprolol OR bosentan OR "candesartan cilexetil" OR captopril OR carteolol OR carvedilol OR chlorthalidone OR clonidine OR cilazapril OR clevidipine OR deserpidine OR diazoxide OR diltiazem OR doxazosin OR enalapril OR enalaprilat OR "eprosartan mesylate" OR hydrochlorothiazide OR felodipine OR fenoldopam OR fosinopril OR guanabenz OR guanadrel OR guanethidine OR guanfacine OR hydralazine OR irbesartan OR isradipine OR labetalol OR lisinopril OR "losartan potassium" OR macitentan OR mecamylamine OR methyldopa OR metoprolol OR metyrosine OR mibefradil OR minoxidil OR moexipril OR moxonidine OR nadolol OR nebivolol OR nicardipine OR nifedipine OR nisoldipine OR nitroprusside OR “olmesartan medoxomil" OR omapatrilat OR penbutolol OR perindopril OR phentolamine OR pindolol OR prazosin OR propranolol OR quinapril OR ramipril OR rescinnamine OR reserpine OR sildenafil OR "sodium nitroprusside" OR tadalafil OR telmisartan OR terazosin OR timolol OR trandolapril OR treprostinil OR trimethaphan OR valsartan OR verapamil OR diuretic OR diuretics OR thiazide OR "adrenergic beta-antagonist" OR "adrenergic beta-antagonists" OR "adrenergic alpha-antagonist" OR "adrenergic alpha-antagonists" OR "Angiotensin-Converting Enzyme Inhibitors"[Mesh] OR "ace inhibitor" OR "ace inhibitors" OR "angiotensin-converting enzyme inhibitor" OR "angiotensin-converting enzyme inhibitors" OR "angiotensin II Receptor Blockers" OR "angiotensin II Receptor Blockers" OR "Angiotensin II Type 2 Receptor Blockers"[Mesh] OR "calcium channel blocker" OR "calcium channel blockers" OR "ganglionic blocker" OR "ganglionic blockers" OR "vasodilator agent" OR "vasodilator agents" OR "Vasodilator Agents"[Mesh] OR nitrates OR "Nitrates"[Mesh] OR nitrites OR "Nitrites"[Mesh]) AND (gwa[ti] OR gwas[ti] OR genome[ti] OR "gene"[ti] OR "genes"[ti] OR "genotype"[ti] OR "genotypes"[ti] OR "genotyping"[ti] OR "snp"[ti] OR "snps"[ti] OR polymorphism*[ti] OR "DNA"[ti] OR allele[ti] OR alleles[ti] OR "minor allele" OR "minor alleles" OR "single nucleotide polymorphism" OR "single nucleotides polymorphisms" OR genetic*[ti] OR "trait locus"[ti] OR "loci"[ti] OR “Genetic Predisposition to Disease"[MeSH] OR "Genotype"[MeSH] OR "Gene Frequency"[MeSH] OR "Polymorphism, Single Nucleotide/genetics"[MESH] OR "Polymorphism, Single Nucleotide"[MAJR] OR “Genetic Loci”[Mesh] OR “Genetic Association Studies"[Mesh] OR “Genetic Variation"[Mesh] OR “Blood Pressure/genetics"[Mesh]) NOT ("review"[pt] OR “Cross-Sectional Studies"[MeSH Terms] OR Comment[pt] OR Editorial[pt] OR Letter[pt] OR "Case Reports"[pt] OR "case control"[ti] OR "case report"[ti] OR "case study"[ti] OR "case series"[ti] OR "Case-Control Studies"[Mesh] OR "Follow-Up Studies"[Mesh] OR "observational study" [ti] OR "prospective cohort" [ti] OR "cohort studies" [Mesh:NoExp] OR "cohort study"[ti] OR "Longitudinal Studies" [Mesh:NoExp] OR "Follow-Up Studies"[mesh] OR “Retrospective Studies"[mesh] OR “non-randomized”[ti] OR "follow up study"[ti] OR "Cross-Sectional Studies" [MeSH Terms] OR "Prospective Studies" [MeSH Terms] OR "epidemiology"[Subheading] OR "pulmonary hypertension" OR "pulmonary arterial hypertension" OR "heart transplant" OR "heart failure" OR "cystic fibrosis" OR "cancer" OR "neoplasms" OR "fibromyalgia" OR “alzheimers" OR “alzheimer" OR "pregnant" OR "pregnancy" OR "obesity/drug therapy" [mesh] OR "diet therapy"[mesh] OR "diet therapy"[subheading] OR "DASH"[tiab] OR meal[ti] OR "nutritional intervention" OR "dietary intervention" OR "nutritional counseling" OR "dietary counseling" OR "caffeine" OR "eating change" OR "activities of daily living" OR "dehydration" OR "dehydrate" OR "dehydrated" OR "dietary salt" OR sodium OR "epilepsy" OR "influenza" OR "flu" OR "pneumonia" OR "septicemia" OR arthritis OR "hiv" OR "Acquired Immunodeficiency Syndrome" OR "meningitis" OR "substance abuse" OR "alcoholism" OR "drug abuse" OR "spinal cord"[ti] OR “Sleep"[Majr] OR “Sleep Apnea Syndromes"[Majr] OR sleep[ti] OR contraceptive*[ti] OR (animals[mesh] NOT humans[mesh]) OR rat[ti] OR rats[ti] OR mouse[ti] OR mice[ti] OR pig[ti] OR pigs[ti] OR dog[ti] OR dogs[ti] OR canine[ti] OR cow[ti] OR cows[ti] OR bovine[ti]).
} 
Table 4. Criteria 4a: Genes $(n=27)$ in major blood pressure regulatory pathways associated with physical activity and/or obesity in genome wide association studies. The search was conducted on 05/11/2017 and a total of 188 records were identified.

\begin{abstract}
("mean arterial" OR "blood pressure"[mesh] OR "blood pressure" OR "blood pressures" OR "arterial pressure" OR "arterial pressures" OR hypertension OR hypotension OR normotension OR hypertensive OR hypotensive OR normotensive OR "systolic pressure" OR "diastolic pressure" OR "pulse pressure" OR "venous pressure" OR "pressure monitor" OR hypotension OR "pre hypertension" OR "bp response" OR “bp decrease" OR "bp reduction" OR "bp monitor" OR "bp monitors" OR "bp measurement" OR "Blood Pressure/genetics" [MeSH]) AND ("resting blood pressure" OR "resting BP" OR "exercise"[mesh] OR exercise[ti] OR exercises OR running[mesh] OR running[ti] OR "bicycle" OR "bicycles" OR "bicycling" OR walking OR walking[mesh] OR walking[ti] OR treadmill* OR "weight lifting" OR "weight training" OR "weight bearing" OR "resistance training" OR "strength training" OR "endurance training" OR "speed training" OR "training duration" OR "training frequency" OR "training intensity" OR "aerobic endurance" OR "aerobic training" OR "physical activity" OR "motor activity"[mesh] OR Overweight OR “Overweight"[Mesh] OR BMI OR “body mass" OR “Body Mass Index"[MeSH] OR “Waist Circumference"[MeSH] OR obesity OR “Obesity"[Mesh] OR obese) AND (gwa OR gwas OR "Genetic Association Studies"[Mesh] OR genomewide OR "genome wide" OR "genomewide association" OR "genome wide association" OR "genome-wide interaction") NOT ("review" [pt] OR “Cross-Sectional Studies"[MeSH Terms] OR Comment[pt] OR Editorial[pt] OR Letter[pt] OR “Case Reports"[pt] OR "case control" [ti] OR "case report"[ti] OR “case study"[ti] OR "case series"[ti] OR “Case-Control Studies"[Mesh] OR "Follow-Up Studies"[Mesh] OR "observational study"[ti] OR "prospective cohort"[ti] OR "cohort studies" [Mesh:NoExp] OR "cohort study"[ti] OR “Longitudinal Studies" [Mesh:NoExp] OR "Follow-Up Studies"[mesh] OR "Retrospective Studies"[mesh] OR "non-randomized"[ti] OR "follow up study"[ti] OR "Cross-Sectional Studies"[MeSH Terms] OR "Prospective Studies"[MeSH Terms] OR "epidemiology"[Subheading] OR "pulmonary hypertension" OR "pulmonary arterial hypertension" OR "heart transplant" OR "heart failure" OR "cystic fibrosis" OR "cancer" OR "neoplasms" OR "fibromyalgia" OR "alzheimers" OR "alzheimer" OR "pregnant" OR "pregnancy" OR "obesity/drug therapy"[mesh] OR "diet therapy"[mesh] OR "diet therapy"[subheading] OR "DASH"[tiab] OR meal[ti] OR "nutritional intervention" OR "dietary intervention" OR "nutritional counseling" OR "dietary counseling" OR "caffeine" OR "eating change" OR "activities of daily living" OR "dehydration" OR "dehydrate" OR "dehydrated" OR "dietary salt" OR sodium OR "epilepsy" OR "influenza" OR "flu" OR "pneumonia" OR "septicemia" OR arthritis OR "hiv" OR "Acquired Immunodeficiency Syndrome" OR "meningitis" OR "substance abuse" OR "alcoholism" OR “drug abuse" OR "spinal cord"[ti] OR “Sleep"[Majr] OR "Sleep Apnea Syndromes"[Majr] OR sleep[ti] OR contraceptive*[ti] OR (animals[mesh] NOT humans[mesh]) OR rat[ti] OR rats[ti] OR mouse[ti] OR mice[ti] OR pig[ti] OR pigs[ti] OR dog[ti] OR dogs[ti] OR canine[ti] OR cow[ti] OR cows[ti] OR bovine[ti]).
\end{abstract}

Potential reports were screened by PP for title and abstract, and by PP and LSP for full text review to determine if they qualified. In addition, the authors performed manual searches of reference lists from relevant reports in their files for possible inclusion. Any questions regarding qualification were resolved by discussion by PP and LSP.

\title{
3. Results
}

The search yielded 1408 reports plus 14 records obtained from manual searches of the authors' files. Of these, 57 trials qualified. Please see Figure 1 for the flowchart detailing the systematic search of potential reports and selection process for the reports that qualified. Of the qualifying trials containing human genes in major BP regulatory pathways, we extracted 11 genes associated with the BP response to exercise under criteria 1 [33-40]; 18 genes associated with hypertension in GWAS under criteria 2 [41-48]; 28 genes associated with the BP response to pharmacotherapy under criteria 3 [49-70]; 27 genes associated with physical activity and/or obesity in GWAS under criteria 4a [71-85]; and 29 genes associated with BP, physical activity, and/or obesity in non-GWAS under criteria 4b [86-88] displayed in Supplemental Digital Content (SDC) 1 Table S1. In addition, we included the 41 genes from our preliminary work that were reported to be associated with hypertension or the BP response to exercise or pharmacotherapy [27-29].

See SDC 1 Table S1 for the complete signature of 154 prioritized BP and exercise genes by our predetermined inclusion criteria. 


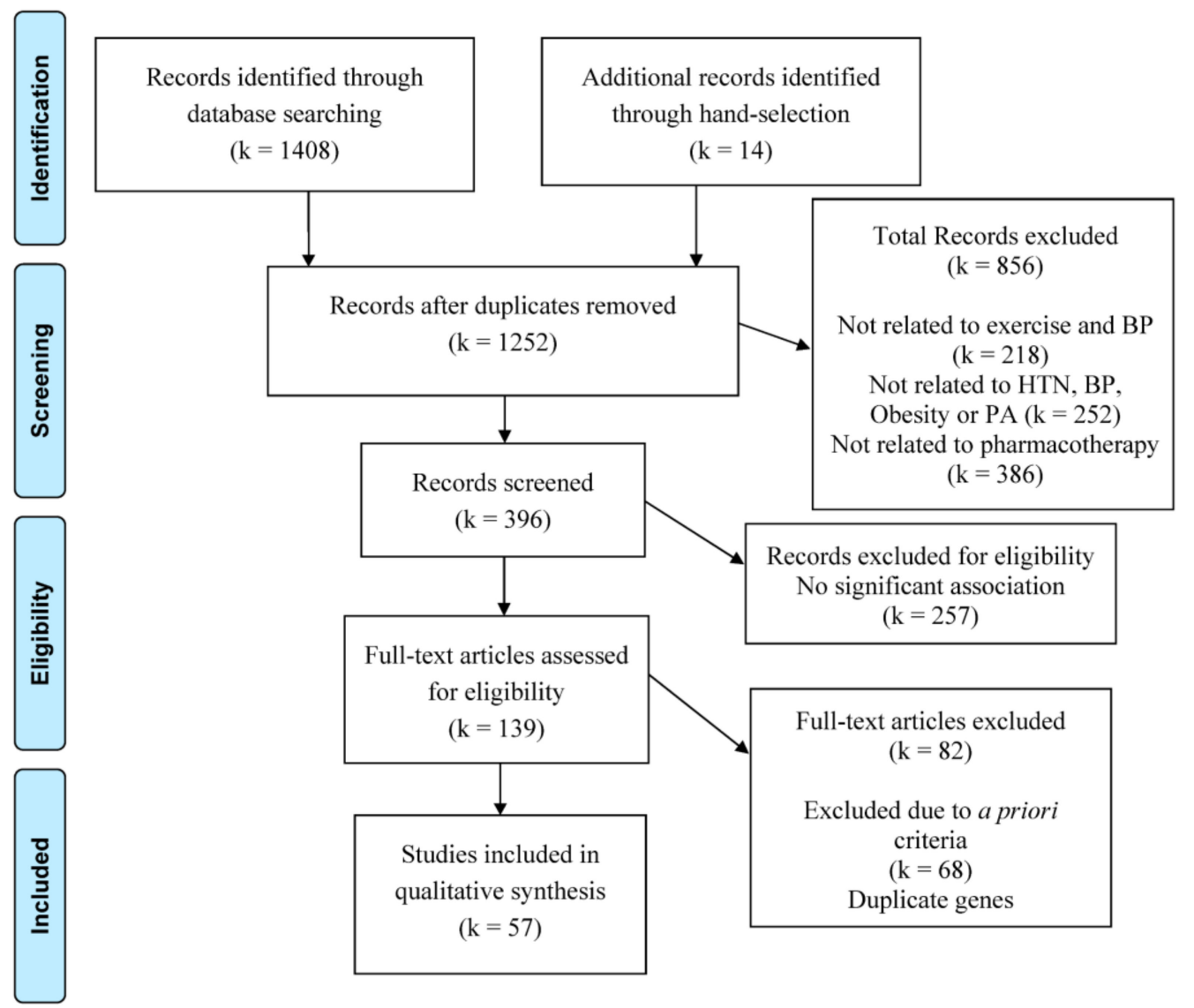

Figure 1. The systematic search trial selection process. BP: blood pressure; HTN: hypertension; PA: physical activity.

\section{Discussion}

The AHA/ACC rated exercise as one of the best lifestyle therapeutic approaches to prevent and treat hypertension because aerobic exercise training lowers BP 5-8 $\mathrm{mmHg}$ among adults with hypertension [3]. Nearly 20 years ago, the HERITAGE study investigators established that the BP response to exercise was heritable. This finding set off investigator-initiated studies examining the genetic basis for the antihypertensive effects of exercise using a candidate gene approach, as this was the technology available at that time. Despite the best intentions and efforts of these research teams, these candidate gene studies were met with little success $[17,25,26]$. The failure of the candidate gene approach is best illustrated by a systematic review we recently completed on the influence of the angiotensin converting enzyme (ACE) insertion/deletion polymorphism rs4340 on human endurance exercise performance and cardiovascular health [89]. ACE rs4340 is the most widely investigated genetic variant in the exercise genomic literature. Despite the extensive volume of literature on $A C E$ rs4340, we concluded that due to disparate findings no definitive conclusions could be made regarding the role of $A C E$ rs 4340 on endurance exercise performance or the BP response to exercise.

More recently, using newer genomic technology that emerged since our discovery phase candidate gene association studies, in a replication cohort we deep-sequenced the exons of genes contained on a prioritized signature of 41 genes identified from our earlier work combined with those from a systematic review of the literature of genes reported to be associated with hypertension or the BP response to exercise or pharmacotherapy using the Illumina TruSeq Custom Amplicon kit [27-29]. Even after adjustment for multiple testing, we found that $61 \%$ of the genes in the prioritized panel associated with the BP response to exercise. Clinical features such as resting BP, age, gender, and cardiometabolic biomarkers explained $66 \%-92 \%$ of the variation in the BP response to exercise. Yet, the genetic variants 
that emerged from these analyses explained $2 \%-15 \%$ of the variance in the BP response to exercise, a magnitude that is larger than typically reported in exercise genomic studies $[25,90]$.

We attributed the high proportion of significant BP-genotype associations that we found to the following methodological strategies we instituted [27-29]: a randomized control repeated measure design with subjects who were their own control; a focused inquiry of variants with a prioritized panel of genes obtained from a systematic review of the literature that reduced the search space within the genome; high throughput exon sequencing on functional gene regions; standardized protocols that included a closely monitored, well-controlled exercise exposure; and adjustment for multiple testing based on genetic variants exhibiting variability in the number of minor alleles and with unique genotypic values. This series of studies are proof of concept that a focused genomic inquiry on functional portions of genes based upon a systematic review of the literature is a time and cost-efficient way to investigate the genomic basis for the antihypertensive effects of exercise. See Figure 2 for a conceptual overview of this systematic review approach to investigate the genomics of the antihypertensive effects of exercise. However, future investigator-initiated studies remain to be done expanding upon this approach among a large ethnically and gender diverse sample of adults with hypertension to continue to gain information about the genomic basis for the antihypertensive effects of exercise with the long-term goal of fine-tuning exercise prescriptions to optimize the BP benefits of exercise.

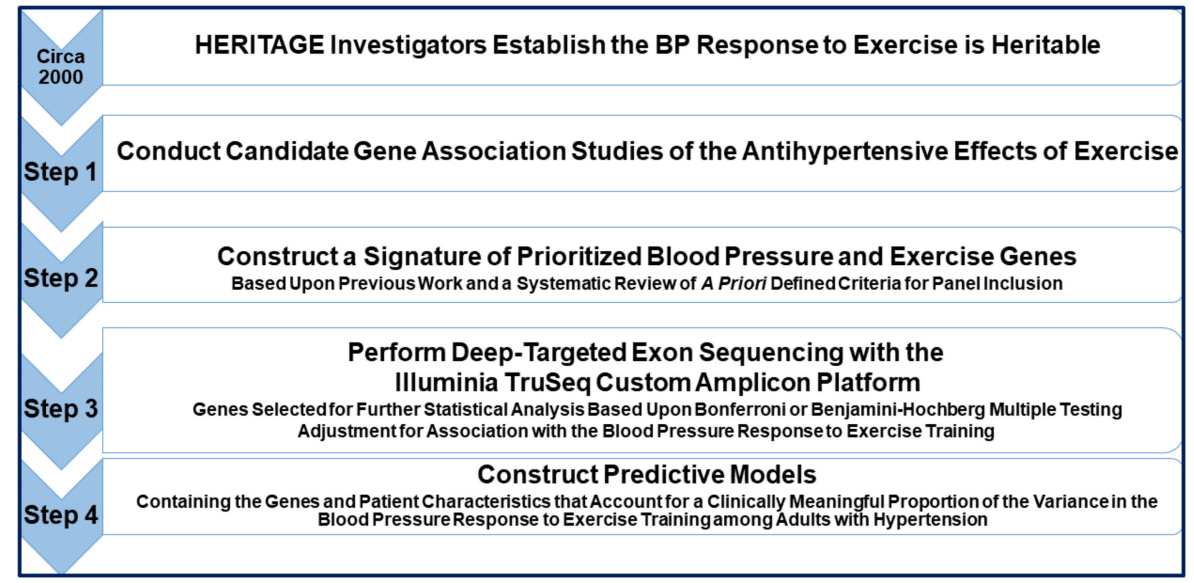

Figure 2. A Systematic review approach to assemble a targeted gene panel to study the genomics of the antihypertensive effects of exercise. HERITAGE: HEalth, RIsk Factors Exercise TrAining and Genetics; BP: blood pressure.

We acknowledge a limitation of our approach is that there are more sophisticated system analyses and bioinformatic approaches now being used with GWAS, human disease, and pathway data sets such as ENCODE and NHGRI GWAS that integrate omic high throughput technology of the genome, transcriptome, proteome, and epigenome to elucidate the genomic basis for hypertension (See SDC 1 Table 1 criteria 4a). In fact, we used such data sets in our most recent work to derive regulatory elements for the polymorphisms that passed multiple testing threshold for significant associations with the BP to exercise [27-29]. Another limitation is that genes have transcription-factor and post-transcriptional regulators so that RNA expression levels could also be used to find signatures associated with the antihypertensive effects of exercise as opposed to BP and exercise genes per se. Genes have epistatic and pleiotropic effects further complicating the identification of genetic variants that explain a clinically meaningful proportion of the BP response to exercise. Presently no data set repositories exist for genes that have been reported to associated with the BP response to exercise that have passed preestablished thresholds for multiple testing. Considering the importance of hypertension as a public health problem and the critical role exercise has in the treatment of hypertension, we posit that the methods we have presented in this systematic review represent a time and cost-efficient approach to construct targeted 
gene signatures whose exons can be deep-sequenced to gain insight into the genomic basis for the antihypertensive effects of exercise. Furthermore, these methods could easily be adapted to design prioritized signatures of the transcriptome, proteome, and epigenome as they regulate the BP response to exercise.

Supplementary Materials: The following are available online at http://www.mdpi.com/2073-4425/10/4/295/s1, Supplemental Digital Content 1. Table S1. The Prioritized Panel of 154 Blood Pressure Genes from a Systematic Review of the Literature by the Four A Priori Criteria Combined with Our Preliminary Work.

Funding: The University of Connecticut (UConn) Research Foundation; UConn Institute for Health, Intervention, and Policy; Connecticut Institute for Clinical and Translational Science.

Acknowledgments: The Jackson Laboratory's Scientific Services (Genome Technologies) for performing the deep-targeting exon sequencing and preparation of the variant calling files. L.S.P. contributed to study design conceptualization, data collection, statistical analyses, results interpretation, manuscript writing and reviewing, and acquisition of funding; Elizabeth D. Schifano contributed to statistical analyses, results interpretation, and manuscript writing and reviewing; Garrett I. Ash contributed to study design conceptualization, data collection, statistical analyses, results interpretation, manuscript reviewing, and acquisition of funding; Gregory A. Panza contributed to data collection, statistical analyses, results interpretation, and manuscript writing and reviewing; Lauren ML Corso contributed to data collection and manuscript writing and reviewing; Ming-Hui Chen contributed to statistical analyses, results interpretation, and manuscript reviewing; Ved Deshpande contributed to statistical analyses, results interpretation, and manuscript reviewing; Amanda Zaleski contributed to data collection, manuscript writing and reviewing, and acquisition of funding; Burak Cilhoroz contributed to statistical analyses, results interpretation, and manuscript writing and reviewing; Paulo Farinatti contributed to data collection, manuscript writing and reviewing, and acquisition of funding; B.A.T. contributed to data collection, manuscript writing and reviewing, and acquisition of funding; Rachel J. O'Neill contributed to results interpretation and manuscript writing and reviewing; and Paul D. Thompson contributed to data collection and manuscript writing and reviewing.

Conflicts of Interest: The authors declare no conflict of interest.

\section{References}

1. Benjamin, E.J.; Muntner, P.; Alonso, A.; Bittencourt, M.S.; Callaway, C.W.; Carson, A.P.; Chamberlain, A.M.; Chang, A.R.; Cheng, S.; Das, S.R.; et al. Heart Disease and Stroke Statistics-2019 Update: A Report from the American Heart Association. Circulation 2019, 139, e56-e528. [CrossRef] [PubMed]

2. Fisher, N.D.L.; Curfman, G. Hypertension-A Public Health Challenge of Global Proportions. JAMA 2018, 320, 1757-1759. [CrossRef] [PubMed]

3. Whelton, P.K.; Carey, R.M.; Aronow, W.S.; Casey, D.E., Jr.; Collins, K.J.; Dennison Himmelfarb, C.; DePalma, S.M.; Gidding, S.; Jamerson, K.A.; Jones, D.W.; et al. 2017 ACC/AHA/AAPA/ABC/ACPM/AGS/ APhA/ASH/ASPC/NMA/PCNA Guideline for the Prevention, Detection, Evaluation, and Management of High Blood Pressure in Adults: A Report of the American College of Cardiology/American Heart Association Task Force on Clinical Practice Guidelines. Hypertension 2018, 71, e127-e248.

4. Naci, H.; Salcher-Konrad, M.; Dias, S.; Blum, M.R.; Anova, S.; Nunan, D.; Ioannidis, J. How Does Exercise Treatment Compare with Antihypertensive Medications? A Network Meta-Analysis of 391 RCTs Assessing Exercise and Medication Effects on Systolic Blood Pressure. Br. J. Sports Med. 2018. [CrossRef] [PubMed]

5. Pescatello, L.S. Exercise Measures Up to Medication as Antihypertensive Therapy: Its Value has Long been Underestimated. Br. J. Sports Med. 2018. [CrossRef]

6. Chobanian, A.V.; Bakris, G.L.; Black, H.R.; Cushman, W.C.; Green, L.A.; Izzo, J.L., Jr.; Jones, D.W.; Materson, B.J.; Oparil, S.; Wright, J.T., Jr.; et al. Seventh Report of the Joint National Committee on Prevention, Detection, Evaluation, and Treatment of High Blood Pressure. Hypertension 2003, 42, 1206-1252. [CrossRef] [PubMed]

7. Law, M.R.; Morris, J.K.; Wald, N.J. Use of Blood Pressure Lowering Drugs in the Prevention of Cardiovascular Disease: Meta-Analysis of 147 Randomised Trials in the Context of Expectations from Prospective Epidemiological Studies. BMJ 2009, 338, b1665. [CrossRef] [PubMed]

8. Whelton, P.K.; He, J.; Appel, L.J.; Cutler, J.A.; Havas, S.; Kotchen, T.A.; Roccella, E.J; Stout, R.; Vallbona, C.; Winston, M.C.; et al. Primary Prevention of Hypertension: Clinical and Public Health Advisory from the National High Blood Pressure Education Program. JAMA 2002, 288, 1882-1888. [CrossRef] [PubMed] 
9. Physical Activity Guidelines Advisory Committee. 2018 Physical Activity Guidelines Advisory Committee Scientific Report; Department of Health and Human Services: Washington, DC, USA, 2018.

10. Powell, K.E.; King, A.C.; Buchner, D.M.; Campbell, W.W.; DiPietro, L.; Erickson, K.I.; Hillman, C.H.; Jakicic, J.M.; Janz, K.F.; Katzmarzyk, P.T.; et al. The Scientific Foundation for the Physical Activity Guidelines for Americans, 2nd Edition. J. Phys. Act. Health 2018, 16, 1-11. [CrossRef] [PubMed]

11. Pescatello, L.S.; MacDonald, H.V.; Ash, G.I.; Lamberti, L.M.; Farquhar, W.B.; Arena, R.; Johnson, B.T. Assessing the Existing Professional Exercise Recommendations for Hypertension: A Review and Recommendations for Future Research Priorities. Mayo Clin. Proc. 2015, 90, 801-812. [CrossRef] [PubMed]

12. Kim, H.; Andrade, F.C.D. Diagnostic Status and Age at Diagnosis of Hypertension on Adherence to Lifestyle Recommendations. Prev. Med. Rep. 2018, 13, 52-56. [CrossRef] [PubMed]

13. Mu, L.; Cohen, A.J.; Mukamal, K.J. Prevalence and Predictors of Resistance and Aerobic Exercise among Hypertensive Adults in the United States. J. Hum. Hypertens. 2015, 29, 394-395. [CrossRef] [PubMed]

14. Bouchard, C.; Rankinen, T. Individual Differences in Response to Regular Physical Activity. Med. Sci. Sports Exerc. 2001, 33, S446-S451. [CrossRef] [PubMed]

15. Bouchard, C.; Blair, S.N.; Church, T.S.; Earnest, C.P.; Hagberg, J.M.; Hakkinen, K.; Jenkins, N.T.; Karavirta, L.; Kraus, W.E.; Leon, A.S.; et al. Adverse Metabolic Response to Regular Exercise: Is it a Rare Or Common Occurrence? PLoS ONE 2012, 7, e37887. [CrossRef] [PubMed]

16. Rankinen, T.; Gagnon, J.; Perusse, L.; Chagnon, Y.C.; Rice, T.; Leon, A.S.; Skinner, J.S.; Wilmore, J.H.; Rao, D.C.; Bouchard, C. AGT M235T and ACE ID Polymorphisms and Exercise Blood Pressure in the HERITAGE Family Study. Am. J. Physiol. Heart Circ. Physiol. 2000, 279, H368-H374. [CrossRef] [PubMed]

17. Rankinen, T.; Roth, S.M.; Bray, M.S.; Loos, R.; Perusse, L.; Wolfarth, B.; Hagberg, J.M.; Bouchard, C. Advances in Exercise, Fitness, and Performance Genomics. Med. Sci. Sports Exerc. 2010, 42, 835-846. [CrossRef]

18. Rankinen, T.; Rice, T.; Perusse, L.; Chagnon, Y.C.; Gagnon, J.; Leon, A.S.; Skinner, J.S.; Wilmore, J.H.; Rao, D.C.; Bouchard, C. NOS3 Glu298Asp Genotype and Blood Pressure Response to Endurance Training: The HERITAGE Family Study. Hypertension 2000, 36, 885-889. [CrossRef] [PubMed]

19. Rankinen, T.; Church, T.; Rice, T.; Markward, N.; Leon, A.S.; Rao, D.C.; Skinner, J.S.; Blair, S.N.; Bouchard, C. Effect of Endothelin 1 Genotype on Blood Pressure is Dependent on Physical Activity Or Fitness Levels. Hypertension 2007, 50, 1120-1125. [CrossRef]

20. Augeri, A.L.; Tsongalis, G.J.; van Heest, J.L.; Maresh, C.M.; Thompson, P.D.; Pescatello, L.S. The Endothelial Nitric Oxide Synthase -786 T>C Polymorphism and the Exercise-Induced Blood Pressure and Nitric Oxide Responses among Men with Elevated Blood Pressure. Atherosclerosis 2009, 204, e28-e34. [CrossRef]

21. Ash, G.I.; Eicher, J.D.; Pescatello, L.S. The Promises and Challenges of the use of Genomics in the Prescription of Exercise for Hypertension: The 2013 Update. Curr. Hypertens. Rev. 2013, 9, 130-147. [CrossRef]

22. Blanchard, B.E.; Tsongalis, G.J.; Guidry, M.A.; LaBelle, L.A.; Poulin, M.; Taylor, A.L.; Maresh, C.M.; Devaney, J.; Thompson, P.D.; Pescatello, L.S. RAAS Polymorphisms Alter the Acute Blood Pressure Response to Aerobic Exercise among Men with Hypertension. Eur. J. Appl. Physiol. 2006, 97, 26-33. [CrossRef]

23. Pescatello, L.S.; Blanchard, B.E.; Tsongalis, G.J.; Maresh, C.M.; O'Connell, A.; Thompson, P.D. The $\alpha$-Adducin Gly460Trp Polymorphism and the Antihypertensive Effects of Exercise among Men with High Blood Pressure. Clin. Sci. (Lond.) 2007, 113, 251-258. [CrossRef] [PubMed]

24. Pescatello, L.S.; Blanchard, B.E.; van Heest, J.L.; Maresh, C.M.; Gordish-Dressman, H.; Thompson, P.D. The Metabolic Syndrome and the Immediate Antihypertensive Effects of Aerobic Exercise: A Randomized Control Design. BMC Cardiovasc. Disord. 2008, 8, 12. [CrossRef] [PubMed]

25. Bruneau, M.L., Jr.; Johnson, B.T.; Huedo-Medina, T.B.; Larson, K.A.; Ash, G.I.; Pescatello, L.S. The Blood Pressure Response to Acute and Chronic Aerobic Exercise: A Meta-Analysis of Candidate Gene Association Studies. J. Sci. Med. Sport 2016, 19, 424-431. [CrossRef] [PubMed]

26. Bouchard, C. Overcoming Barriers to Progress in Exercise Genomics. Exerc. Sport Sci. Rev. 2011, 39, $212-217$. [CrossRef]

27. Cilhoroz, B.T.; Schifano, E.D.; Panza, G.A.; Ash, G.I.; Corso, L.; Chen, M.H.; Deshpande, V.; Zaleski, A.; Farinatti, P.; Santos, L.P.; et al. FURIN Variant Associations with Postexercise Hypotension are Intensity and Race Dependent. Physiol. Rep. 2019, 7, e13952. [CrossRef]

28. Pescatello, L.S.; Shifano, E.D.; Ash, G.I.; Panza, G.A.; Lamberti, L.; Chen, M.; Deshpande, V.; Zaleski, A.; Farinatti, P.; Taylor, B.A.; et al. Deep-Targeted Exon Sequencing Reveals Renal Polymorphisms Associate with Postexercise Hypotension among African Americans. Physiol. Rep. 2016, 4, e12992. [CrossRef] 
29. Pescatello, L.S.; Schifano, E.D.; Ash, G.I.; Panza, G.A.; Corso, L.M.L.; Chen, M.H.; Deshpande, V.; Zaleski, A.; Cilhoroz, B.; Farinatti, P.; et al. Deep-Targeted Sequencing of Endothelial Nitric Oxide Synthase Gene Exons Uncovers Exercise Intensity and Ethnicity-Dependent Associations with Post-Exercise Hypotension. Physiol. Rep. 2017, 5. [CrossRef]

30. Moher, D.; Liberati, A.; Tetzlaff, J.; Altman, D.G. PRISMA Group. Preferred Reporting Items for Systematic Reviews and Meta-Analyses: The PRISMA Statement. J. Clin. Epidemiol. 2009, 62, 1006-1012. [CrossRef]

31. Moher, D.; Shamseer, L.; Clarke, M.; Ghersi, D.; Liberati, A.; Petticrew, M.; Shekelle, P.; Stewart, L.A.; PRISMA-P Group. Preferred Reporting Items for Systematic Review and Meta-Analysis Protocols (PRISMA-P) 2015 Statement. Syst. Rev. 2015, 4, 1. [CrossRef] [PubMed]

32. Lavie, C.J.; de Schutter, A.; Parto, P.; Jahangir, E.; Kokkinos, P.; Ortega, F.B.; Arena, R.; Milani, R.V. Obesity and Prevalence of Cardiovascular Diseases and Prognosis-the Obesity Paradox Updated. Prog. Cardiovasc. Dis. 2016, 58, 537-547. [CrossRef] [PubMed]

33. Deschamps, C.L.; Connors, K.E.; Klein, M.S.; Johnsen, V.L.; Shearer, J.; Vogel, H.J.; Devaney, J.M.; Gordish-Dressman, H.; Many, G.M.; Barfield, W. The ACTN3 R577X Polymorphism is Associated with Cardiometabolic Fitness in Healthy Young Adults. PLoS ONE 2015, 10, e0130644. [CrossRef] [PubMed]

34. Franks, P.W.; Bhattacharyya, S.; Luan, J.; Montague, C.; Brennand, J.; Challis, B.; Brage, S.; Ekelund, U.; Middelberg, R.P.; O'Rahilly, S.; et al. Association between Physical Activity and Blood Pressure is Modified by Variants in the G-Protein Coupled Receptor 10. Hypertension 2004, 43, 224-228. [CrossRef]

35. Heradien, M.; Revera, M.; van der Merwe, L.; Goosen, A.; Corfield, V.A.; Brink, P.A.; Mayosi, B.M.; Moolman-Smook, J.C. Abnormal Blood Pressure Response to Exercise Occurs More Frequently in Hypertrophic Cardiomyopathy Patients with the R92W Troponin T Mutation than in those with Myosin Mutations. Heart Rhythm 2009, 6, S18-S24. [CrossRef] [PubMed]

36. Jayewardene, A.F.; Mavros, Y.; Gwinn, T.; Hancock, D.P.; Rooney, K.B. Associations between CD36 Gene Polymorphisms and Metabolic Response to a Short-Term Endurance-Training Program in a Young-Adult Population. Appl. Physiol. Nutr. Metab. 2015, 41, 157-167. [CrossRef]

37. Masuki, S.; Mori, M.; Tabara, Y.; Miki, T.; Sakurai, A.; Morikawa, M.; Miyagawa, K.; Higuchi, K.; Nose, H.; Shinshu University Genetic Research Consortium. Vasopressin V1a Receptor Polymorphism and Interval Walking Training Effects in Middle-Aged and Older People. Hypertension 2010, 55, 747-754. [CrossRef]

38. Nunes, R.A.B.; Barroso, L.P.; da Costa Pereira, A.; Krieger, J.E.; Mansur, A.J. Gender-Related Associations of Genetic Polymorphisms of $\alpha$-Adrenergic Receptors, Endothelial Nitric Oxide Synthase and Bradykinin B2 Receptor with Treadmill Exercise Test Responses. Open Heart 2014, 1, e000132. [CrossRef] [PubMed]

39. Rivera, M.A.; Echegaray, M.; Rankinen, T.; Pérusse, L.; Rice, T.; Gagnon, J.; Leon, A.S.; Skinner, J.S.; Wilmore, J.H.; Rao, D. Angiogenin Gene-Race Interaction for Resting and Exercise BP Phenotypes: The HERITAGE Family Study. J. Appl. Physiol. 2001, 90, 1232-1238. [CrossRef] [PubMed]

40. Shiwaku, K.; Nogi, A.; Anuurad, E.; Kitajima, K.; Enkhmaa, B.; Shimono, K.; Yamane, Y. Difficulty in Losing Weight by Behavioral Intervention for Women with Trp64Arg Polymorphism of the $\beta$ 3-Adrenergic Receptor Gene. Int. J. Obes. 2003, 27, 1028. [CrossRef] [PubMed]

41. Fujimaki, T.; Oguri, M.; Horibe, H.; Kato, K.; Matsuoka, R.; Abe, S.; Tokoro, F.; Arai, M.; Noda, T.; Watanabe, S. Association of a Transcription Factor 21 Gene Polymorphism with Hypertension. Biomed. Rep. 2015, 3, 118-122. [CrossRef] [PubMed]

42. Huan, T.; Esko, T.; Peters, M.J.; Pilling, L.C.; Schramm, K.; Schurmann, C.; Chen, B.H.; Liu, C.; Joehanes, R.; Johnson, A.D. A Meta-Analysis of Gene Expression Signatures of Blood Pressure and Hypertension. PLoS Genet. 2015, 11, e1005035. [CrossRef]

43. Lu, X.; Wang, L.; Lin, X.; Huang, J.; Charles Gu, C.; He, M.; Shen, H.; He, J.; Zhu, J.; Li, H. Genome-Wide Association Study in Chinese Identifies Novel Loci for Blood Pressure and Hypertension. Hum. Mol. Genet. 2014, 24, 865-874. [CrossRef] [PubMed]

44. Murakata, Y.; Fujimaki, T.; Yamada, Y. Association of a Butyrophilin, Subfamily 2, Member A1 Gene Polymorphism with Hypertension. Biomed. Rep. 2014, 2, 818-822. [CrossRef] [PubMed]

45. Ong, K.L.; Li, M.; Tso, A.W.; Xu, A.; Cherny, S.S.; Sham, P.C.; Tse, H.F.; Lam, T.H.; Cheung, B.M.; Lam, K.S.; et al. Association of Genetic Variants in the Adiponectin Gene with Adiponectin Level and Hypertension in Hong Kong Chinese. Eur. J. Endocrinol. 2010, 163, 251-257. [CrossRef]

46. Rong, S.; Zhou, X.; Wang, Z.; Wang, X.; Wang, Y.; Xue, C.; Li, B. Glutathione S-Transferase M1 and T1 Polymorphisms and Hypertension Risk: An Updated Meta-Analysis. J. Hum. Hypertens. 2018, 1. [CrossRef] 
47. Zhang, K.; Rao, F.; Miramontes-Gonzalez, J.P.; Hightower, C.M.; Vaught, B.; Chen, Y.; Greenwood, T.A.; Schork, A.J.; Wang, L.; Mahata, M. Neuropeptide Y (NPY): Genetic Variation in the Human Promoter Alters Glucocorticoid Signaling, Yielding Increased NPY Secretion and Stress Responses. J. Am. Coll. Cardiol. 2012, 60, 1678-1689. [CrossRef]

48. Zhang, W.; Wang, H.; Guan, X.; Niu, Q.; Li, W. Variant rs2237892 of KCNQ1 is Potentially Associated with Hypertension and Macrovascular Complications in Type 2 Diabetes Mellitus in a Chinese Han Population. Genom. Proteom. Bioinform. 2015, 13, 364-370. [CrossRef]

49. Chen, Y.; Liu, D.; Zhang, P.; Zhong, J.; Zhang, C.; Wu, S.; Zhang, Y.; Liu, G.; He, M.; Jin, L. Impact of ACE2 Gene Polymorphism on Antihypertensive Efficacy of ACE Inhibitors. J. Hum. Hypertens. 2016, 30, 766. [CrossRef]

50. Bruck, H.; Schwerdtfeger, T.; Toliat, M.; Leineweber, K.; Heusch, G.; Philipp, T.; Nürnberg, P.; Brodde, O. Presynaptic $\alpha-2 \mathrm{C}$ Adrenoceptor-mediated Control of Noradrenaline Release in Humans: Genotype-or Age-Dependent? Clin. Pharmacol. Ther. 2007, 82, 525-530. [CrossRef]

51. Chen, G.; Jiang, S.; Mao, G.; Zhang, S.; Hong, X.; Tang, G.; Li, Z.; Liu, X.; Zhang, Y.; Xing, H. CYP2C9 Ile359Leu Polymorphism, Plasma Irbesartan Concentration and Acute Blood Pressure Reductions in Response to Irbesartan Treatment in Chinese Hypertensive Patients. Methods Find. Exp. Clin. Pharmacol. 2006, 28, 19-24.

52. Chen, W.; Shu, Y.; Li, Q.; Xu, L.; Roederer, M.W.; Fan, L.; Wu, L.; He, F.; Luo, J.; Tan, Z. Polymorphism of ORM1 is Associated with the Pharmacokinetics of Telmisartan. PLoS ONE 2013, 8, e70341. [CrossRef]

53. Anthony, E.G.; Richard, E.; Lipkowitz, M.S.; Kelley, S.T.; Alcaraz, J.E.; Shaffer, R.A.; Bhatnagar, V. Association of Phosphodiesterase 4 Polymorphism (rs702553) with Blood Pressure in the African American Study of Kidney Disease and Hypertension Genomics Study. Pharm. Genom. 2013, 23, 442-444. [CrossRef] [PubMed]

54. Donner, K.M.; Hiltunen, T.P.; Hannila-Handelberg, T.; Suonsyrjä, T.; Kontula, K. STK39 Variation Predicts the Ambulatory Blood Pressure Response to Losartan in Hypertensive Men. Hypertens. Res. 2012, 35, 107. [CrossRef] [PubMed]

55. Duarte, J.D.; Turner, S.T.; Tran, B.; Chapman, A.B.; Bailey, K.R.; Gong, Y.; Gums, J.G.; Langaee, T.Y.; Beitelshees, A.L.; Cooper-Dehoff, R.M. Association of Chromosome 12 Locus with Antihypertensive Response to Hydrochlorothiazide may Involve Differential YEATS4 Expression. Pharm. J. 2013, $13,257$. [CrossRef] [PubMed]

56. Frau, F.; Zaninello, R.; Salvi, E.; Ortu, M.F.; Braga, D.; Velayutham, D.; Argiolas, G.; Fresu, G.; Troffa, C.; Bulla, E. Genome-Wide Association Study Identifies CAMKID Variants Involved in Blood Pressure Response to Losartan: The SOPHIA Study. Pharmacogenomics 2014, 15, 1643-1652. [CrossRef] [PubMed]

57. Duan, R.; Cui, W.; Wang, H. Association of the Antihypertensive Response of Iptakalim with KCNJ11 (Kir6. 2 Gene) Polymorphisms in Chinese Han Hypertensive Patients. Acta Pharmacol. Sin. 2011, 32, 1078. [CrossRef]

58. Bhatnagar, V.; O'connor, D.T.; Brophy, V.H.; Schork, N.J.; Richard, E.; Salem, R.M.; Nievergelt, C.M.; Bakris, G.L.; Middleton, J.P.; Norris, K.C. G-Protein-Coupled Receptor Kinase 4 Polymorphisms and Blood Pressure Response to Metoprolol among African Americans: Sex-Specificity and Interactions. Am. J. Hypertens. 2009, 22, 332-338. [CrossRef]

59. Kamide, K.; Asayama, K.; Katsuya, T.; Ohkubo, T.; Hirose, T.; Inoue, R.; Metoki, H.; Kikuya, M.; Obara, T.; Hanada, H. Genome-Wide Response to Antihypertensive Medication using Home Blood Pressure Measurements: A Pilot Study Nested within the HOMED-BP Study. Pharmacogenomics 2013, 14, 1709-1721. [CrossRef]

60. Kamide, K.; Yang, J.; Matayoshi, T.; Takiuchi, S.; Horio, T.; Yoshii, M.; Miwa, Y.; Yasuda, H.; Yoshihara, F.; Nakamura, S. Genetic Polymorphisms of L-Type Calcium Channel $\alpha 1 C$ and $\alpha 1 \mathrm{D}$ Subunit Genes are Associated with Sensitivity to the Antihypertensive Effects of L-Type Dihydropyridine Calcium-Channel Blockers. Circ. J. 2009, 73, 732-740. [CrossRef]

61. Konoshita, T.; Kato, N.; Fuchs, S.; Mizuno, S.; Aoyama, C.; Motomura, M.; Makino, Y.; Wakahara, S.; Inoki, I.; Miyamori, I.; et al. Genetic Variant of the Renin-Angiotensin System and Diabetes Influences Blood Pressure Response to Angiotensin Receptor Blockers. Diabetes Care 2009, 32, 1485-1490. [CrossRef]

62. He, F.; Luo, J.; Luo, Z.; Fan, L.; He, Y.; Zhu, D.; Gao, J.; Deng, S.; Wang, Y.; Qian, Y. The KCNH2 Genetic Polymorphism $(1956, \mathrm{C}>\mathrm{T})$ is a Novel Biomarker that is Associated with CCB and $\alpha, \beta$-ADR Blocker Response in EH Patients in China. PLoS ONE 2013, 8, e61317. [CrossRef] 
63. He, F.; Luo, J.; Zhang, Z.; Luo, Z.; Fan, L.; He, Y.; Wen, J.; Zhu, D.; Gao, J.; Wang, Y. The RGS2 (-391, C> G) Genetic Variation Correlates to Antihypertensive Drug Responses in Chinese Patients with Essential Hypertension. PLOS ONE 2015, 10, e0121483. [CrossRef]

64. Hong, X.; Xing, H.; Yu, Y.; Wen, Y.; Zhang, Y.; Zhang, S.; Tang, G.; Xu, X. Genetic Polymorphisms of the Urea Transporter Gene are Associated with Antihypertensive Response to Nifedipine GITS. Methods Find. Exp. Clin. Pharmacol. 2007, 29, 3-10. [CrossRef]

65. Guo, R.; Chen, L.; Li, L.; Guo, X.; Sun, J.; Xiong, X.; Cheng, Z.; Li, Y.; Chen, X. Association of GSTM1 Null Polymorphism with Isosorbide-5-Mononitrate Cardiovascular Response and Involvement of CGRP in Healthy Chinese Male Volunteers. Pharm. Genom. 2011, 21, 142-151. [CrossRef]

66. Geshi, E.; Kimura, T.; Yoshimura, M.; Suzuki, H.; Koba, S.; Sakai, T.; Saito, T.; Koga, A.; Muramatsu, M.; Katagiri, T. A Single Nucleotide Polymorphism in the Carboxylesterase Gene is Associated with the Responsiveness to Imidapril Medication and the Promoter Activity. Hypertens. Res. 2005, 28, 719. [CrossRef]

67. Jia, J.; Men, C.; Tang, K.; Zhan, Y. Apelin Polymorphism Predicts Blood Pressure Response to Losartan in Older Chinese Women with Essential Hypertension. Genet. Mol. Res. 2015, 14, 6561-6568. [CrossRef] [PubMed]

68. Lanzani, C.; Citterio, L.; Glorioso, N.; Manunta, P.; Tripodi, G.; Salvi, E.; Carpini, S.D.; Ferrandi, M.; Messaggio, E.; Staessen, J.A.; et al. Adducin- and Ouabain-Related Gene Variants Predict the Antihypertensive Activity of Rostafuroxin, Part 2: Clinical Studies. Sci. Transl. Med. 2010, 2, 59ra87. [CrossRef] [PubMed]

69. Zhang, Y.; Zhang, M.; Niu, T.; Xu, X.; Zhu, G.; Huo, Y.; Chen, C.; Wang, X.; Xing, H.; Peng, S. D919G Polymorphism of Methionine Synthase Gene is Associated with Blood Pressure Response to Benazepril in Chinese Hypertensive Patients. J. Hum. Genet. 2004, 49, 296. [CrossRef]

70. Zhang, Y.; Hong, X.; Xing, H.; Li, J.; Yong, H.; Xu, X. E112D Polymorphism in the Prolylcarboxypeptidase Gene is Associated with Blood Pressure Response to Benazepril in Chinese Hypertensive Patients. Chin. Med. J. 2009, 122, 2461-2465. [PubMed]

71. Andreassen, O.A.; Djurovic, S.; Thompson, W.K.; Schork, A.J.; Kendler, K.S.; O’Donovan, M.C.; Rujescu, D.; Werge, T.; van de Bunt, M.; Morris, A.P. Improved Detection of Common Variants Associated with Schizophrenia by Leveraging Pleiotropy with Cardiovascular-Disease Risk Factors. Am. J. Hum. Genet. 2013, 92, 197-209. [CrossRef]

72. Flaquer, A.; Baumbach, C.; Kriebel, J.; Meitinger, T.; Peters, A.; Waldenberger, M.; Grallert, H.; Strauch, K. Mitochondrial Genetic Variants Identified to be Associated with BMI in Adults. PLoS ONE 2014, 9, e105116. [CrossRef] [PubMed]

73. Flores-Alfaro, E.; Fernández-Tilapa, G.; Salazar-Martínez, E.; Cruz, M.; Illades-Aguiar, B.; Parra-Rojas, I. Common Variants in the CRP Gene are Associated with Serum C-Reactive Protein Levels and Body Mass Index in Healthy Individuals in Mexico. Genet. Mol. Res. 2012, 11, 2258. [CrossRef]

74. Hotta, K.; Kitamoto, A.; Kitamoto, T.; Mizusawa, S.; Teranishi, H.; Matsuo, T.; Nakata, Y.; Hyogo, H.; Ochi, H.; Nakamura, T; et al. Genetic Variations in the CYP17A1 and NT5C2 Genes are Associated with a Reduction in Visceral and Subcutaneous Fat Areas in Japanese Women. J. Hum. Genet. 2012, 57, 46-51. [CrossRef]

75. Hager, J.; Dina, C.; Francke, S.; Dubois, S.; Houari, M.; Vatin, V.; Vaillant, E.; Lorentz, N.; Basdevant, A.; Clement, K. A Genome-Wide Scan for Human Obesity Genes Reveals a Major Susceptibility Locus on Chromosome 10. Nat. Genet. 1998, 20, 304. [CrossRef]

76. Kaess, B.M.; Barnes, T.A.; Stark, K.; Charchar, F.J.; Waterworth, D.; Song, K.; Wang, W.Y.; Vollenweider, P.; Waeber, G.; Mooser, V. FGF21 Signalling Pathway and Metabolic traits-genetic Association Analysis. Eur. J. Hum. Genet. 2010, 18, 1344. [CrossRef] [PubMed]

77. Kong, X.; Zhang, X.; Xing, X.; Zhang, B.; Hong, J.; Yang, W. The Association of Type 2 Diabetes Loci Identified in Genome-Wide Association Studies with Metabolic Syndrome and its Components in a Chinese Population with Type 2 Diabetes. PLoS ONE 2015, 10, e0143607. [CrossRef] [PubMed]

78. Kim, Y.K.; Kim, Y.; Hwang, M.Y.; Shimokawa, K.; Won, S.; Kato, N.; Tabara, Y.; Yokota, M.; Han, B.; Lee, J.H. Identification of a Genetic Variant at 2q12. 1 Associated with Blood Pressure in East-Asians by Genome-Wide Scan Including Gene-Environment Interactions. BMC Med. Genet. 2014, 15, 65. [CrossRef] [PubMed]

79. Muiya, N.P.; Wakil, S.; Al-Najai, M.; Tahir, A.I.; Baz, B.; Andres, E.; Al-Boudari, O.; Al-Tassan, N.; Al-Shahid, M.; Meyer, B.F. A Study of the Role of GATA2 Gene Polymorphism in Coronary Artery Disease Risk Traits. Gene 2014, 544, 152-158. [CrossRef] [PubMed] 
80. Sull, J.W.; Yang, S.J.; Kim, S.; Jee, S.H. The ABCG2 Polymorphism rs 2725220 is Associated with Hyperuricemia in the Korean Population. Genom. Inform. 2014, 12, 231-235. [CrossRef] [PubMed]

81. Teixeira, A.A.; Quinto, B.M.R.; Dalboni, M.A.; Rodrigues, C.J.D.O.; Batista, M.C. Association of IL-6 Polymorphism-174G/C and Metabolic Syndrome in Hypertensive Patients. BioMed Res. Int. 2015, 2015, 927589. [CrossRef] [PubMed]

82. Wang, M.; Li, J.; Yeung, V.; Zee, B.; Yu, R.; Ho, S.; Waye, M. Four Pairs of gene-gene Interactions Associated with Increased Risk for Type 2 Diabetes (CDKN2BAS-KCNJ11), Obesity (SLC2A9-IGF2BP2, FTO-APOA5), and Hypertension (MC4R-IGF2BP2) in Chinese Women. Meta Gene 2014, 2, 384-391. [CrossRef]

83. Wang, Y.; Bos, S.D.; Harbo, H.F.; Thompson, W.K.; Schork, A.J.; Bettella, F.; Witoelar, A.; Lie, B.A.; Li, W.; McEvoy, L.K. Genetic Overlap between Multiple Sclerosis and several Cardiovascular Disease Risk Factors. Mult. Scler. J. 2016, 22, 1783-1793. [CrossRef] [PubMed]

84. Wei, F.; Cai, C.; Yu, P.; Lv, J.; Ling, C.; Shi, W.; Jiao, H.; Chang, B.; Yang, F.; Tian, Y. Quantitative Candidate Gene Association Studies of Metabolic Traits in Han Chinese Type 2 Diabetes Patients. Genet. Mol. Res. 2015, 14, 15471-15481. [CrossRef] [PubMed]

85. Zeller, T.; Wild, P.; Szymczak, S.; Rotival, M.; Schillert, A.; Castagne, R.; Maouche, S.; Germain, M.; Lackner, K.; Rossmann, H.; et al. Genetics and Beyond-the Transcriptome of Human Monocytes and Disease Susceptibility. PLoS ONE 2010, 5, e10693. [CrossRef] [PubMed]

86. Akintunde, A.; Nondi, J.; Gogo, K.; Jones, E.S.; Rayner, B.L.; Hackam, D.G.; Spence, J.D. Physiological Phenotyping for Personalized Therapy of Uncontrolled Hypertension in Africa. Am. J. Hypertens. 2017, 30, 923-930. [CrossRef]

87. Ehret, G.B.; Ferreira, T.; Chasman, D.I.; Jackson, A.U.; Schmidt, E.M.; Johnson, T.; Thorleifsson, G.; Luan, J.; Donnelly, L.A.; Kanoni, S.; et al. The Genetics of Blood Pressure Regulation and its Target Organs from Association Studies in 342,415 Individuals. Nat. Genet. 2016, 48, 1171-1184. [CrossRef]

88. Wahl, S.; Drong, A.; Lehne, B.; Loh, M.; Scott, W.R.; Kunze, S.; Tsai, P.; Ried, J.S.; Zhang, W.; Yang, Y. Epigenome-Wide Association Study of Body Mass Index, and the Adverse Outcomes of Adiposity. Nature 2017, 541, 81. [CrossRef] [PubMed]

89. Pescatello, L.S.; Corso, L.M.L.; Santos, L.P.; Livingston, J.; Taylor, B.A. Chapter 21 Angiotensin Converting Enzyme and the Genomics of Endurance Performance. In Routledge Handbook of Sport and Exercise Systems Genetics, 1st ed.; Lightfoot, J.T., Hubal, M.J., Roth, S.M., Eds.; Routledge Taylor and France Group: London, UK, 2019.

90. Pescatello, L.S.; Devaney, J.M.; Hubal, M.J.; Thompson, P.D.; Hoffman, E.P. Highlights from the Functional Single Nucleotide Polymorphisms Associated with Human Muscle Size and Strength Or FAMuSS Study. Biomed. Res. Int. 2013, 2013, 643575. [CrossRef] [PubMed]

(C) 2019 by the authors. Licensee MDPI, Basel, Switzerland. This article is an open access article distributed under the terms and conditions of the Creative Commons Attribution (CC BY) license (http://creativecommons.org/licenses/by/4.0/). 